\title{
Incidencia de retinopatía del prematuro en pacientes producto de embarazos múltiples concebidos en forma natural y mediante técnicas de reproducción asistida
}

\section{Incidence of retinopathy of prematurity in multiple pregnancies conceived naturally and by assisted-reproduction techniques}

\author{
Luis P. Orozco-Gómez*, Irving Flores-Carmona, Leonor Hernández-Salazar, Karlina Minguez-Lorenzo, \\ Karla I. Rojas-Morales y Juan C. Medina-Díaz
}

Centro Médico Nacional "20 de Noviembre", ISSSTE, Ciudad de México, México

\begin{abstract}
Resumen
Objetivo: Determinar la incidencia de la retinopatía del prematuro (ROP) en productos obtenidos de embarazos múltiples concebidos de forma natural y mediante técnicas de reproducción asistida, en el Centro Médico Nacional "20 de Noviembre" del Instituto de Seguridad y Servicios Sociales de los Trabajadores del Estado, durante el periodo de junio de 2008 a junio de 2016. Método: Observacional, comparativo, descriptivo y retrospectivo. El grupo 1 constituido por niños obtenidos por técnicas de reproducción asistida, y el grupo 2 constituido por niños obtenidos por fecundación natural. El protocolo del estudio fue evaluado y aceptado por los comités de ética en investigación y bioseguridad del Centro Médico Nacional "20 de Noviembre"con número de folio 497.2017. Resultados: Se estudiaron 184 pacientes. El grupo 1 (reproducción asistida) con 90 pacientes, el grupo 2 (fecundación natural) con 94 pacientes. La edad media gestacional para el grupo 1 fue de 32.7 semanas, con una desviación de 2.12 semanas; para el grupo 2, la edad media de gestación fue de 32.9 semanas, con una desviación de 2.17 semanas. El peso promedio del grupo 1 fue de 1,673 g (o 425) y del grupo 2 fue de 1,784 $\mathrm{g}$ ( $\sigma$ 491). En el grupo 1 se diagnosticaron 5 pacientes con ROP (5/90 5.6\%]) y en el grupo 2, 8 pacientes (8/94 8.5\%]). Conclusiones: Si bien se advierte que un embarazo múltiple incrementa la posibilidad de prematuridad y/o bajo peso al nacer y con ello mayores probabilidades de desarrollar ROP, en el estudio se observó que no existe diferencia en el riesgo relacionado con la técnica de concepción, sea reproducción asistida o fecundación natural para el embarazo múltiple. Sin embargo, sí se observó una asociación positiva para embarazos múltiples de más de dos productos y en pacientes con peso extremadamente bajo al nacer.
\end{abstract}

Palabras clave: Reproducción asistida. Embarazo múltiple. Retinopatía del prematuro.

\section{Abstract}

Objective: To determine the incidence of retinopathy of prematurity in multiple pregnancies conceived naturally and by assisted-reproduction techniques at the Centro Médico Nacional "20 de Noviembre" del Instituto de Seguridad y Servicios Sociales de los Trabajadores del Estado, from June 2008 to June 2016. Method: Observational, comparative, descriptive and

Correspondencia:

*Luis P. Orozco-Gómez

Félix Cuevas, 540

Col. Del Valle, Del. Benito Juárez $\quad$ Fecha de recepción: 08-03-2018

Disponible en internet: 01-05-2019

Ciudad de México, México

Fecha de recepción: 08-03-2018

Rev Mex Oftalmol. 2019;93(3):117-124

E-mail: Iporozco@issste.gob.mx

DOI: 10.24875/RMO.M19000066

www.rmo.com.mx

0187-4519/๑ 2018 Sociedad Mexicana de Oftalmología. Publicado por Permanyer México. Este es un artículo Open Access bajo la licencia CC BY-NC-ND (http://creativecommons.org/licenses/by-nc-nd/4.0/). 
retrospective study. Group 1 consisted of children conceived by assisted-reproduction techniques and Group 2 consisted of naturally conceived children. The protocol was revised and accepted by the research ethics and biosafety committees of the Centro Médico Nacional "20 de Noviembre" with file number 497.2017. Results: One hundred and eighty-four patients were included. Group 1 consisted of 90 patients (assisted reproduction) and Group 2 consisted of 94 patients. Mean gestational age for Group 1 was 32.7 weeks \pm 2.12 weeks and for group 2 was 32.9 weeks \pm 2.17 weeks. Average weight for group 1 was $1,673 \mathrm{~g}(\sigma \mathrm{\sigma} 425)$ and for group 2 was $1,784 \mathrm{~g}(\sigma \mathrm{4} 41)$. There were 5 patients with retinopathy of prematurity in group 1 (5/90) and 8 patients in group 2 (8/94). Conclusions: Although it is known that multiple pregnancies increase the risk of prematurity and/or low birth weight, hence increasing the probability of developing retinopathy of prematurity, this study did not find risk differences based on the type of conception for multiple pregnancies. However, a positive association was observed for multiple pregnancies of more than two fetuses and in patients with extremely low birth weight.

Key words: Assisted reproduction. Multiple pregnancy. Retinopathy of prematurity.

\section{Introducción}

La incidencia de nacimientos múltiples ha aumentado constantemente en los últimos 30 años, principalmente debido al uso creciente de tecnologías de reproducción asistida y al aumento en la edad promedio materna de la concepción. Las técnicas de reproducción asistida se definen como cualquier procedimiento que implique el manejo tanto de óvulos como de espermatozoides, o de embriones, con el fin de establecer un embarazo ya sea por transferencia intrafalopiana de gametos, fertilización in vitro o inyección intracitoplasmática de esperma ${ }^{1}$. Los embarazos múltiples se asocian con un mayor riesgo de complicaciones que los embarazos únicos, tanto para la madre como para los bebés.

En el embarazo múltiple, los productos tienen un mayor riesgo de restricción del crecimiento intrauterino. En el Reino Unido, la Autoridad de Fertilización y Embriología Humana informó que la mitad de los gemelos (naturales y por reproducción asistida) nacen prematuramente ${ }^{2}$.

Además de las complicaciones generales del embarazo gemelar per se, los embarazos gemelares por técnicas de reproducción asistida generalmente tienen factores adicionales, que incluyen edad materna avanzada, nuliparidad, medicamentos para la fertilidad e intervenciones asociadas, que pueden representar factores de riesgo potenciales para una mayor morbilidad perinatal ${ }^{3}$.

La retinopatía del prematuro (ROP) es una enfermedad ocular provocada por una alteración de la vasculogénesis de la retina, que puede llevar a su desarrollo anormal y a la pérdida total o parcial de la visión, y el factor de riesgo más importante para el desarrollo de la misma es la inmadurez, así como el bajo peso al nacer. Otros factores de riesgo incluyen el suplemento y duración de la exposición al oxígeno, la presencia de sepsis, hemorragia intraventricular y acidosis metabólica.
La prematuridad implica una falta de desarrollo de los vasos de la retina, ya que la vasculogénesis normal se inicia en la semana 16 de vida intrauterina y su desarrollo se alcanza a las 36 semanas en el lado nasal y a las 40 semanas en el lado temporal. Asimismo, si el prematuro es expuesto a concentraciones elevadas de $\mathrm{O}_{2}$ en etapas neonatales tempranas, se inhibe la producción del factor de crecimiento vascular endotelial. Como consecuencia, se retrasa el crecimiento vascular en la retina inmadura, con constricción vascular. Esto resulta en una suspensión de la vascularización normal, con vasoconstricción e isquemia de la retina. La isquemia prolongada produce un aumento en la producción del factor de crecimiento vascular endotelial (VEGF). Si el área de retina inmadura es pequeña, el VEGF podría estimular el crecimiento de vasos normales en el área avascular en el mejor de los casos; pero si el área inmadura es grande, el VEGF estimulará la formación de anastomosis arteriovenosas en el borde entre el área vascularizada y la no vascularizada. Posteriormente los niveles aumentados de VEGF provocaran neovascularización, dilatación y tortuosidad vascular e incluso, rubeosis iridis, con las consecuencias de proliferación fibrovascular, cicatrización y desprendimiento de retina.

Se ha especulado acerca de la relación entre la utilización de técnicas de reproducción asistida y el desarrollo de ROP, o su gravedad, por el aumento de prematuridad y bajo peso al nacer, y algunos estudios han encontrado un aumento en la tasa del deterioro de la visión en niños con bajo peso al nacer. El presente estudio busca revisar la incidencia de ROP en prematuros producto de embarazos múltiples atendidos en el Centro Médico Nacional "20 de Noviembre" del Instituto de Seguridad y Servicios Sociales de los Trabajadores del Estado (ISSSTE), y su relación con la reproducción asistida. 


\section{Material y métodos}

Se realizó una revisión retrospectiva de expedientes de recién nacidos prematuros producto de embarazos múltiples atendidos en el Centro Médico Nacional "20 de Noviembre" del ISSSTE durante el período de junio de 2008 a junio de 2016. Si bien el centro médico se encuentra en la Ciudad de México, es un hospital de concentración que recibe pacientes referidos de todo el país, por lo que el estudio no se circunscribe a esta ciudad. Dado que se valoraron todos los recién nacidos prematuros productos de embarazo múltiple referidos al Servicio de Oftalmología para tamizaje, no fueron incluidos los casos de prematuridad producto de embarazo único, que no son motivo del presente estudio. Tampoco fueron incluidos los productos a término de embarazos múltiples, ya que el estudio se enfoca a la ROP.

Los criterios de inclusión utilizados fueron: pacientes recién nacidos prematuros ${ }^{4}$, es decir, menores de 37 semanas de gestación, independientemente del peso al nacer, producto de embarazos múltiples, atendidos en unidades del ISSSTE y enviados a tamizaje al Servicio de Oftalmología del Centro Médico Nacional "20 de Noviembre", dentro del periodo comprendido entre enero del 2008 a junio de 2016, y que contaran con datos completos en su historia clínica. Se excluyen los pacientes fallecidos en el parto múltiple, pero sí se incluyeron en el estudio los sobrevivientes.

Los datos personales de los pacientes fueron enmascarados para evitar su identificación y con ello proteger su confidencialidad.

De la revisión de expedientes se obtuvo la siguiente información: tipo de fecundación, edad gestacional, tipo de producto, vía de nacimiento, peso al nacer, sexo, presencia de fallecimiento en alguno de los productos, presencia de ROP, estadio de la enfermedad y tratamiento instaurado. La población se dividió en dos grupos, en el grupo 1 se concentraron los recién nacidos de embarazos múltiples obtenidos mediante reproducción asistida, independientemente del método utilizado; en el grupo 2, los recién nacidos productos de embarazos múltiples obtenidos mediante fecundación natural. Los datos obtenidos fueron consignados en un formato diseñado para tal propósito. Se realizó un análisis estadístico de los resultados utilizando la descripción de datos mediante medidas de resumen: frecuencia, moda, media, mediana, rango, desviación estándar. Asimismo, y dado que el estudio se enmarca dentro de la investigación de prevalencia y asociación cruzada, se aplicaron estadísticas y pruebas de análisis de riesgo y asociación: riesgo relativo, riesgo atribuible y razón de momios. En los casos en que alguna frecuencia resultó en cero, se hizo la corrección correspondiente con la adición de 0.5 en todas las frecuencias. Para la organización y cálculos de los datos se utilizó la aplicación de hojas de cálculo Microsoft Excel de Microsoft Office.

\section{Resultados}

Se estudiaron 184 pacientes en total. El grupo 1, constituido por los recién nacidos obtenidos con reproducción asistida, estuvo integrado por 90 pacientes, de los cuales 46 pertenecen al género femenino y 44 al género masculino, y 68 pacientes fueron producto de embarazo de gemelos, 18 de embarazo de trillizos y 4 de embarazo de cuatrillizos. El grupo 2, que concentró los recién nacidos obtenidos por reproducción natural, estuvo constituido por 94 pacientes, 54 del género femenino y 40 del género masculino; 80 pacientes de este grupo fueron gemelos y 14 fueron producto de embarazo de trillizos (Tabla 1). Se excluyó solamente un recién nacido fallecido, producto de embarazo de trillizos, del cual no se contó, por lo tanto, con expediente clínico.

La edad media gestacional para el grupo 1 fue de 32.7 semanas, con una desviación de 2.12 semanas. Para el grupo 2, la edad media de gestación fue de 32.9 semanas, con una desviación de 2.17 semanas. El peso promedio del grupo 1 fue de $1,673 \mathrm{~g}(\sigma 425)$ y del grupo 2 fue de $1,784 \mathrm{~g}$ ( $\sigma$ 491). Todos los pacientes de ambos grupos fueron obtenidos mediante intervención por cesárea (parto abdominal), de acuerdo a la indicación institucional para embarazo múltiple. En el grupo 1 (Tabla 2) se diagnosticaron 5 pacientes con ROP (5/90), de los cuales, 1 paciente se encontró en estadio I, 1, en estadio II, 2, en estadio III, y 1, en estadio IV. Recibieron tratamiento 3 pacientes de este grupo, 1 con monoterapia mediante inyección intravítrea de antiangiogénico y los otros 2 fueron tratados mediante terapia combinada de antiangiogénico más aplicación de láser transpupilar.

En el grupo 2, 8 pacientes presentaron ROP, uno de ellos en estadio II, y los otros 7 desarrollaron estadio III; todos recibieron tratamiento, 2 de ellos con monoterapia mediante inyección intravítrea de antiangiogénico, y 6 pacientes fueron tratados mediante terapia combinada de antiangiogénico más aplicación de láser transpupilar.

Ningún paciente presentó secuelas graves atribuidas a la ROP.

La exposición al riesgo, considerada en este estudio por la reproducción asistida como factor de exposición, 
Tabla 1. Recién nacidos prematuros, producto de embarazos múltiples, enviados a tamizaje de retinopatía del prematuro, según sexo, tipo de parto y tipo de reproducción (Centro Médico Nacional "20 de Noviembre", Instituto de Seguridad y Servicios Sociales para los Trabajadores del Estado, 2008-2016.)

\begin{tabular}{|c|c|c|c|c|c|c|}
\hline \multirow[t]{2}{*}{ Tipo de reproducción } & \multicolumn{2}{|c|}{ Sexo } & \multicolumn{3}{|c|}{ Tipo de parto } & \multirow[t]{2}{*}{ Total } \\
\hline & Femenino & Masculino & Gemelar & Trillizos & Cuatrillizos & \\
\hline Reproducción asistida & 46 & 44 & 68 & 18 & 4 & 90 \\
\hline Reproducción natural & 54 & 40 & 80 & 14 & 0 & 94 \\
\hline Total & 100 & 84 & 148 & 22 & 4 & 184 \\
\hline
\end{tabular}

Tabla 2. Recién nacidos prematuros, producto de embarazos múltiples, enviados a tamizaje de ROP, según diagnóstico de retinopatía del prematuro y tipo de reproducción (Centro Médico Nacional "20 de Noviembre", Instituto de Seguridad y Servicios Sociales para los Trabajadores del Estado, 2008-2016.)

\begin{tabular}{|l|c|c|c|c|c|c|}
\hline & \multicolumn{4}{|c|}{ Con retinopatía del recién nacido prematuro } & Sin ROP \\
\cline { 2 - 8 } & Grado I & Grado II & Grado III & Grado IV & Total \\
\hline Reproducción asistida & 1 & 1 & 2 & 1 & 5 & 85 \\
\hline Reproducción natural & 0 & 1 & 7 & 1 & 13 & 171 \\
\hline Total & 1 & 2 & 9 & 1 & 86 & 13 \\
\hline
\end{tabular}

ROP: retinopatía del prematuro.

fue de 90 pacientes, con un riesgo de 5/90. En el grupo de referencia (grupo 2) el riesgo fue de 8/94 (Tabla 3). Al aplicar análisis de asociación y riesgo, se encontró un riesgo relativo de 0.65 , por lo que se concluye que no hay asociación entre el tipo de reproducción y la presencia del padecimiento (ROP). El análisis de riesgo atribuible confirma dicha conclusión con un valor negativo (-0.03), es decir, que no influyó el factor de riesgo con la aparición de la enfermedad. Al aplicar la razón de momios se obtuvo un resultado de 0.63 , por lo que el tamaño del efecto también confirma la no asociación del padecimiento con la exposición al factor de riesgo analizado, con una probabilidad de 0.39 (39\%) de que ocurriera la enfermedad en los recién nacidos producto de reproducción asistida (considerando 50\% la ocurrencia al azar).

Se realizó también el análisis de asociación y riesgo para los grupos, de acuerdo al tipo de parto, gemelar y de tres o más productos. No se encontró asociación para el grupo de parto gemelar (Tabla 4); sin embargo, sí se encontró un riesgo relativo de 1.95 para el grupo de pacientes de parto múltiple de tres o más productos, por lo que se puede concluir que hay una asociación positiva entre la reproducción asistida y la presencia de ROP, por la mayor probabilidad de producir embarazos múltiples de más productos (Tabla 5). En este análisis se observó que 3 de cada 100 pacientes expuestos al factor de riesgo desarrollarían ROP (riesgos atribuible de 0.03 ), con una razón de momios de 2.02 , que representa una probabilidad del $67 \%$ de desarrollar la enfermedad (17\% más que el azar).

Si bien el peso al nacer no estaba considerado como elemento del presente estudio para el desarrollo de ROP, se realizó el análisis de riesgo de reproducción asistida en los grupos de recién nacidos con bajo peso al nacer (menor de 2,500 g), muy bajo peso al nacer (menor de $1,500 \mathrm{~g}$ ) y peso al nacer extremadamente bajo (menor de 1,000 g). Los resultados se consignan en las tablas 6, 7 y 8. No se encontró asociación en los grupos de recién nacidos de bajo y muy bajo peso al nacer. Sin embargo, sí se observó una asociación positiva entre la reproducción asistida y el desarrollo de ROP en los bebés de peso al nacer extremadamente bajo (riesgo relativo de 1.2), con un riesgo atribuible de 6 para cada 100 pacientes expuestos y una razón de momios de 1.33 , es decir, de una probabilidad de ocurrencia de $57 \%$ ( $7 \%$ más que el azar).

Considerando la inmadurez como factor causal de la retinopatía, se analizó el riesgo y asociación de la reproducción asistida en los grupos de recién nacidos de acuerdo a su edad gestacional, tanto para muy prematuros (28 a 32 semanas de gestación) como para prematuros extremos (menores de 28 semanas de gestación), ver tabla 9 y 10. No se encontró asociación en 
Tabla 3. Análisis de riesgo de reproducción asistida y ROP

\begin{tabular}{|l|c|c|c|c|}
\hline & ROP sí & ROP no & Total & Riesgo relativo $=0.6527$ \\
\hline Reproducción asistida sí & 5 & 85 & 90 & 94 \\
\hline Reproducción asistida no & 8 & 86 & 184 & Riesgo atribuible $=-0.0295$ \\
\hline
\end{tabular}

ROP: retinopatía del prematuro.

Tabla 4. Análisis de riesgo de la reproducción asistida y ROP en recién nacidos prematuros producto de parto gemelar

\begin{tabular}{|l|c|c|c|c|}
\hline & ROP sí & ROP no & Total & \\
\hline Reproducción asistida sí & 4 & 64 & 68 & Riesgo relativo $=0.5882$ \\
\hline Reproducción asistida no & 8 & 72 & 80 & Riesgo atribuible $=-0.0411$ \\
\hline
\end{tabular}

ROP: retinopatía del prematuro.

Tabla 5. Análisis de riesgo de la reproducción asistida y ROP en recién nacidos prematuros producto de parto de trillizos y cuatrillizos

\begin{tabular}{|l|c|c|c|l|}
\hline & ROP sí & ROP no & Total & \\
\hline Reproducción asistida sí & 1.5 & 21.5 & 23 & Riesgo relativo = 1.9565 \\
\hline Reproducción asistida no & 0.5 & 14.5 & 15 & Riesgo atribuible $=0.0318$ \\
\hline
\end{tabular}

Frecuencias ajustadas.

ROP: retinopatía del prematuro.

Tabla 6. Análisis de riesgo de la reproducción asistida y ROP en recién nacidos prematuros de bajo peso al nacer*

\begin{tabular}{|l|c|c|c|l|}
\hline & ROP sí & ROP no & Total & \\
\hline Reproducción asistida sí & 5 & 85 & 87 & Riesgo relativo $=0.6178$ \\
\hline Reproducción asistida no & 8 & 78 & 86 & Riesgo atribuible $=-0.0355$ \\
\hline
\end{tabular}

*Bajo peso al nacer $=$ menor de $2,500 \mathrm{~g}$

ROP: retinopatía del prematuro.

ninguno de estos grupos, por lo que se puede concluir que no existe un riesgo adicional relacionado con la reproducción asistida para desarrollar ROP en los casos de prematuridad alta y extrema.

\section{Discusión}

La Organización Mundial de la Salud se refiere a la ROP como una de las principales causas de ceguera prevenible a nivel mundial, por lo que el estudio de los factores de riesgo que condicionan su aparición es importante para el diseño de políticas públicas de prevención y detección oportuna. Su frecuencia no es poco considerable en nuestro medio: la Asociación Panamericana de Oftalmología consideró a la ROP como la principal causa de ceguera prevenible en la población infantil latinoamericana, y fue la responsable de aproximadamente 25,000 casos en el $2006^{5}$.

La angiogénesis retiniana se desarrolla en condiciones de hipoxia relativa, por lo tanto, la hiperoxia es tóxica para la retina, especialmente para tejidos inmaduros que aún no desarrollaron sus defensas 
Tabla 7. Análisis de riesgo de la reproducción asistida y ROP en recién nacidos prematuros de muy bajo peso al nacer*

\begin{tabular}{|l|c|c|c|c|}
\hline & ROP sí & ROP no & Total & \\
\hline Reproducción asistida sí & 5 & 28 & 33 & Riesgo relativo $=0.5681$ \\
\hline Reproducción asistida no & 8 & 22 & 30 & Riesgo atribuible $=-0.1151$ \\
\hline
\end{tabular}

*Muy bajo peso al nacer $=$ menor de $1,500 \mathrm{~g}$.

ROP: retinopatía del prematuro.

Tabla 8. Análisis de riesgo de la reproducción asistida y ROP en recién nacidos prematuros de peso al nacer extremadamente bajo*

\begin{tabular}{|l|c|c|c|l|}
\hline & ROP sí & ROP no & Total & \\
\hline Reproducción asistida sí & 2 & 3 & 5 & Riesgo relativo $=1.2000$ \\
\hline Reproducción asistida no & 1 & 2 & 3 & Riesgo atribuible $=0.0666$ \\
\hline
\end{tabular}

*Extremadamente bajo peso al nacer $=$ menor de 1,000 g. ROP: retinopatía del prematuro.

Tabla 9. Análisis de riesgo de la reproducción asistida y ROP en recién nacidos muy prematuros*

\begin{tabular}{|l|c|c|c|c|}
\hline & ROP sí & ROP no & Total & \\
\hline Reproducción asistida sí & 2 & 27 & 29 & Riesgo relativo $=0.3448$ \\
\hline Reproducción asistida no & 6 & 24 & 30 & Riesgo atribuible $=-0.1310$ \\
\hline
\end{tabular}

*Recién nacido muy prematuro = 28 a 32 semanas de gestación

Tabla 10. Análisis de riesgo de la reproducción asistida y ROP en recién nacidos prematuros extremos*

\begin{tabular}{|l|c|c|c|l|}
\hline & ROP sí & ROP no & Total & \\
\hline Reproducción asistida sí & 2.5 & 0.5 & 3 & Riesgo relativo $=1$ \\
\hline Reproducción asistida no & 2.5 & 0.5 & 3 & Riesgo atribuible $=0$ \\
\hline & & N & 6 & Razón de momios $=1$
\end{tabular}

*Prematuridad extrema = Menor de 28 semanas de gestación.

Frecuencias ajustadas

ROP: retinopatía del prematuro.

antioxidantes ${ }^{6}$, lo que hace relevante buscar factores de riesgo que contribuyan a la prematuridad, como es el caso de los embarazos múltiples.

En México, la Secretaria de Salud, en su guía de práctica clínica, y el Grupo ROP México, así como la guía panamericana de detección de ROP, sugieren tamizaje a la siguiente población: todos los recién nacidos pretérmino de $\leq 34$ semanas de edad gestacional y/o $\leq 1,750 \mathrm{~g}$ de peso al nacer; a criterio del médico tratante, los recién nacidos pretérmino $>34$ semanas de gestación y con peso al $>1,750 \mathrm{~g}$ de peso que hayan recibido oxígeno suplementario; y a criterio del médico tratante, los recién nacidos pretérmino que tengan factores de riesgo asociados ${ }^{7}$. Esto establece relevancia para analizar la asociación con otros factores que favorezcan estas condiciones como es el caso de la multiparidad.

Algunos estudios han demostrado que la tasa de deterioro grave de la visión es 26 veces mayor en bebés que pesan $1,500 \mathrm{~g}$ al nacer que en bebés que pesan entre 2,500 y 3,499 g. Los pacientes de embarazos múltiples tienen una mayor tasa de prematuridad 
y bajo peso al nacer que aquellos de embarazos únicos ${ }^{8-13}$.

En 1993, Schaffer, et al..$^{14}$ informaron de una relación entre ROP, bajo peso al nacer y embarazos múltiples, con un $36 \%$ más bajo en bebés nacidos de embarazos únicos que en aquellos de embarazos múltiples.

Estos hallazgos fueron apoyados por Hartikainen-Sorri, et al..$^{15}$. Además, Watts y Adams ${ }^{16}$ encontraron que el $41.6 \%$ de los niños concebidos por fecundación in vitro progresaron al estadio ROP III, en comparación con solo el $9.37 \%$ de los niños concebidos naturalmente.

Los autores especularon que la concepción asistida representa una alta proporción de casos de ROP, especialmente ROP grave $^{17}$.

En el ensayo aleatorizado de Early Treatment for ROP ${ }^{18}$, constituido por 6,998 bebés prematuros que pesaron $<1,251 \mathrm{~g}$ al nacer, el $68 \%$ tenían ROP. Los embarazos únicos representaron el $73 \%$ del grupo con ROP, y no hubo diferencia en las tasas de ROP en estadios I, II y III entre las gestaciones únicas y las gestaciones múltiples. Los autores concluyeron que el desarrollo y la progresión de la ROP es multifactorial $y$, probablemente, involucre factores inherentes al bebé o factores que se transmiten a través de la placenta antes 0 alrededor del momento del nacimiento ${ }^{18}$.

Si bien estos hallazgos establecen una relación importante entre la multiparidad como factor de riesgo para el incremento de la incidencia de prematuridad y, por ende, el mayor riesgo de presentar ROP, no ha sido muy clara la asociación del uso de técnicas de reproducción asistida como factor de riesgo para el incremento de su incidencia.

Los resultados del presente estudio buscan contribuir a este análisis con el fin de aportar elementos a la regulación de pautas sobre el número de embriones a ser implantados en los servicios de reproducción asistida.

\section{Conclusiones}

En el presente estudio se encontró una incidencia de ROP en el $7 \%$ en los recién nacidos prematuros revisados producto de embarazos múltiples, en el 5.5\% entre los producto de embarazos obtenidos con reproducción asistida y en el $8.5 \%$ entre los obtenidos de embarazos múltiples naturales.

No se encontró asociación positiva entre la incidencia de ROP y la reproducción asistida, ni riesgo relativo o atribuible, ni mayor oportunidad de desarrollar la enfermedad por este factor. Sí se encontró asociación entre la reproducción asistida como factor de riesgo para el desarrollo de ROP entre los bebés producto de embarazos con alta multiparidad, y también se identificó un mayor riesgo para los recién nacidos de peso al nacer extremadamente bajo.

El estudio contribuye a advertir que si bien la reproducción asistida per se no necesariamente es un factor de riesgo para el incremento de la incidencia de ROP, sí puede ser un factor de riesgo para los recién nacidos productos de una multiparidad alta y para los productos con un extremo bajo peso al nacer, por lo que deberá establecerse una mayor vigilancia de medidas preventivas en estos dos casos, sobre todo en el uso de oxigenoterapia, para evitar el desarrollo de ROP.

\section{Conflicto de intereses}

Los autores declaran que no recibieron financiación extra para la realización de este trabajo.

\section{Responsabilidades éticas}

Protección de personas y animales. Los autores declaran que para esta investigación no se han realizado experimentos en seres humanos ni en animales.

Confidencialidad de los datos. Los autores declaran que en este artículo no aparecen datos de pacientes.

Derecho a la privacidad y consentimiento informado. Los autores declaran que en este artículo no aparecen datos de pacientes.

\section{Bibliografía}

1. Zuppa A, Scorrano A, Cota F, D'Andrea V, Fracchiolla A, Romagnoli C. Neonatal outcomes in triplet pregnancies: assisted reproduction versus spontaneous conception. J Perinat Med. 2007:35:339e43.

2. Human Fertilization and Embryology Authority. Multiple pregnancies and birth: considering the risks. London: HFEA; 2006.

3. Joy J, McClure I, Cooke E. A comparison of spontaneously conceived twins and twins conceived by artificial reproductive technologies. J Obstet Gynecol. 2008;28:580e5.

4. Organización Mundial de la Salud. Nota descriptiva Nacimientos Prematuros. 19 de febrero de 2018. Disponible en http://www.who.int/es/newsroom/fact-sheets/detail/preterm-birth.

5. Vásquez A. Retinopatía del prematuro, guía clínica diagnóstica-2006. Asociación Panamericana de Oftalmología.

6. Mc Leod DS, Crone SN, Lutty GA. Vasoproliferative in the neonatal dog model of oxygen-induced retinopathy. Invest Ophthalmol. Vis Sci. 1996; 37:1322-33.

7. Retinopatía del prematuro. Grupo ROP México. Disponible en: http:// www.ropmexico.org.mx/archivos/documentos/ROP_Mexico_libro.pdf.

8. Ho SK, Wu PY. Perinatal factors and neonatal morbidity in twin pregnancy. Am J Obstet Gynecol. 1975;122:979-87.

9. Hartikainen-Sorri AL, Kauppila A, Tuimala R, Kovisto M. Factors related to improved outcome for twins. Acta Obstet Gynecol Scand. 1983;62:23-5.

10. McCarthy BJ, Sachs BP, Layde PM, Burton A, Terry JS, Rochat R. The epidemiology of neonatal death in twins. Am J Obstet Gynecol. 1981; 141:252-6.

11. Nylander PP. Perinatal mortality in twins. Acta Genet Med Gemellol. 1979;28:3638

12. Zahalkova M. Perinatal and infant mortality in twins. Prog Clin Biol Res. 1978;24:115-20 
Rev Mex Oftalmol. 2019;93

13. Luke B, Keith LG. The contribution of singletons, twins, and triplets to low birth weight, infant mortality and handicap in the United States. J Reprod Med. 1992;37:661-6.

14. Schaffer DB, Palmer EA, Plotsky DF, Metz HS, Flynn JT, Tung B, et al. Prognostic factors in the natural course of retinopathy of prematurity. Ophthalmology. 1993;100:230-7.

15. Hartikainen-Sorri AL, Kauppila A, Tuimala R, Kovisto M. Factors related to improved outcome for twins. Acta Obstet Gynecol Scand. 1983;62:23-5.
16. Watts $P$, Adams $G$. In vitro fertilization and stage 3 retinopathy of prematurity. Eye. 2000;14:330-3.

17. Bergh T, Ericson A, Hillensjo T, Nygren KG, Wennerholm UB. Deliveries and children born after in-vitro fertilization in Sweden 1982-95: a retrospective cohort study. Lancet. 1999;354:1579-85.

18. Early Treatment for Retinopathy of Prematurity Cooperative Group. Revised indications for the treatment of retinopathy of prematurity. Arch Ophthalmol. 2003;121:1684-96. 